\title{
Dossier : SAVOIRS THÉRAPEUTIQUES ASIATIQUeS ET GLOBALISATION
}

\section{Accentuations et PRAGMATISME}

\author{
Le savoir médical tibétain à \\ destination des étrangers
}

\section{LAURENT PORDIÉ}

Depuis le milieu des années 1970, le développement du tourisme saisonnier dans la région himalayenne du Ladakh attire de nombreux étrangers intéressés par la médecine tibétaine. Quelques praticiens aguerris à l'anglais ont saisi cette occasion pour s'adresser directement à cette nouvelle clientèle au travers de consultations cliniques ou de courts exposés publics. Cet article concerne cette manifestation locale de la globalisation thérapeutique. Plus précisément, il s'intéresse à la façon dont les praticiens présentent un savoir médical élaboré et complexe dans l'intention de le rendre intelligible par des patients internationaux habituellement profanes. Les praticiens procèdent de façon pragmatique en espérant établir un espace de communication mais ni leur savoir médical ni leur pratique ne s'en trouvent profondément modifiés pour autant. La brièveté de ces interactions impose cependant une simplification et une reformulation du savoir qui accentue des caractéristiques existantes en médecine tibétaine, comme le bouddhisme et le " religieux » retenus ici comme exemples, et procure des conceptions parfois erronées aux étrangers. Cet article montre que les processus de "traduction » des connaissances médicales tibétaines ne se réduisent pas à des questions de langage ou à l'influence du nouveau marché de la médecine tibétaine dans la région. Comprendre pleinement ces énonciations du savoir requiert une double exigence supplémentaire : il faut considérer l'architecture du savoir érudit en milieu tibétain et porter attention aux dynamiques sociopolitiques et économiques 
régionales. Ces considérations permettent d'interroger plus généralement certaines idées admises sur l'enchantement et la transformation de la médecine tibétaine dans des contextes similaires.

Mots clés : savoir thérapeutique, globalisation, patients internationaux, traduction, bouddhisme, médecine tibétaine, Ladakh

Autrefois circonscrite à de vastes régions abritant des populations de langues, cultures et religions tibétaines, la médecine savante du Tibet est aujourd'hui devenue un phénomène global. Les logiques de l'économie néolibérale, les politiques favorisant l'exportation et les aspirations renouvelées des praticiens ont contribué à redéfinir les modes de production de cette médecine et à installer définitivement sa présence au niveau international (Adams, 2002 ; Craig et Adams, 2008 ; Millard, 2008 ; Sulek, 2005 ; Vargas, 2008). Ces développements ont également transformé l'offre médicale aux niveaux nationaux. Les versions postsocialistes de l'économie de marché en Mongolie et dans la Région Autonome du Tibet en Chine ont écarté les populations les plus pauvres de la médecine tibétaine (Janes, 1999 et 200I ; Janes et Hilliard, 2008 ; Janes et al., 2005). Ailleurs, la préparation des médicaments et les composés utilisés sont modifiés afin de pouvoir pénétrer les marchés de l'Ouest. C'est ainsi que les cliniques du Tara Institute en Grande-Bretagne ne produisent que des médicaments à base de plantes selon une liste imposée par le gouvernement anglais (Millard, 2008) ou que le mercure, proscrit en Occident pour sa toxicité, est exclu des préparations médicinales dans certains contextes en Asie (Aschoff et Tashigang, 1997 ; Craig, 2006, p. 163). Les exilés tibétains semblent, eux, avoir transformé leur pratique pour répondre aux attentes des patients étrangers, en accentuant particulièrement les aspects de la médecine fondés sur le bouddhisme (Samuel, 200I).

Cette emphase du religieux ou de la spiritualité est également perceptible dans de nombreux contextes similaires. Elle se remarque dans le discours des praticiens et de leurs patients, comme dans les ouvrages et les articles en langues européennes destinés à un public élargi. La conférence d’ouverture du Congrès international de médecine tibétaine tenu à Washington en 1998 constitue à ce titre un exemple éloquent. Elle fut présentée par le célèbre Sogyal Rinpoché'. Ce moine, considéré comme étant la réincarnation d'un maître tibétain, est l'auteur de l'ouvrage The Book of Tibetan Living and Dying (1992), qui connut un remarquable succès international${ }^{2}$. II choisit d'intituler sa communication « Le

I Rinpoché (rin po che) est un titre de révérence donné dans le bouddhisme tibétain pour les toulkou (sprul sku), qui est un terme décrivant certains maîtres qui sont considérés comme ayant la faculté de se réincarner délibérément et avec une parfaite maîtrise pour le bénéfice des êtres.

2 Cet ouvrage est paru en 1993 aux éditions de La Table Ronde, sous le titre Le livre tibétain de la 
cœur spirituel de la médecine tibétaine » et débuta son exposé ainsi : « Ce que je vais entreprendre aujourd'hui, c'est explorer (...) les dimensions spirituelles et mentales du soin dans la tradition bouddhiste tibétaine : je vais parler, sur la base de ma propre expérience, de ce qui fonctionne en Occident » (Rinpoche, 1998). Sogyal Rinpoché annonça immédiatement la nécessaire adaptation de son discours et de son savoir. II contribue très activement à la promotion du bouddhisme tibétain en Occident et sait se rendre efficace auprès d'audiences étrangères. L'orateur ne traita pas spécifiquement de la " science des soins » (sowa rigpa, gso-ba rig-pa ${ }^{3}$ ), la médecine tibétaine savante, mais plus généralement des relations entre le corps et l'esprit dans le bouddhisme de tradition Mahāyāna. L'invitation de cet homme sur le thème précis de la spiritualité dans les arts tibétains de guérir affermit une tendance très contemporaine en Occident, qui consiste à inscrire explicitement la médecine dans le domaine religieux, voire à confondre médecine et bouddhisme. Cette combinaison facilite la pénétration de la médecine tibétaine sur le marché international, en mobilisant tant les réseaux bouddhistes que les partisans des médecines alternatives.

Cet article examine une idée répandue dans les travaux spécialisés portant sur l'interaction contemporaine des médecines d'Asie avec l'Occident ou ses habitants : les médecines sont modulées par le marché et ses besoins et cette transformation concerne tout particulièrement la mise en relief ou l'invention d'aspects ésotériques, religieux ou spirituels (Janes, 2002 ; Langford, 2002 ; Samuel, $200 \mathrm{I}$; Zysk, 200I). Je propose d'examiner dans cette perspective et de façon préliminaire quelques interactions entre thérapeutes et patients internationaux dans la région du Ladakh, aux confins de l'Himalaya indien. Plus précisément, ce texte s'intéresse à la façon dont le savoir médical tibétain est présenté et modulé par les praticiens, ou amchi, dans de telles situations d'interaction.

II s'agit plus précisément de situations interculturelles dans lesquelles praticiens et patients cherchent à adopter les perspectives de l'autre (Gillespie, 2006), en s'efforçant de percevoir les choses à la manière de leurs interlocuteurs. Les amchi adaptent leurs discours à leur auditoire et reproduisent certaines notions communes dans le champ des médecines alternatives en Occident. Ces adaptations et ces appropriations n'ont cependant pas d'incidence notoire sur la transformation du savoir et de la pratique de ces thérapeutes au Ladakh. La démarche des praticiens est pragmatique : ils cherchent à établir un espace de communication. Le savoir des amchi est soumis à de sérieuses contraintes car il faut résumer dans un autre langage et dans le temps réduit d'une consultation ou d'un bref exposé public l'essentiel d'un vaste domaine de connaissances de façon intelligible. Les amchi opèrent un tri puis (re)formulent les éléments choisis de leur savoir. C'est ainsi que la traduction du savoir s'opère, occultant inéluctablement

vie et de la mort.

3 Les termes vernaculaires, tibétains ou ladakhis, sont d'abord transcrits en caractères réguliers (non italiques), tels qu'ils sont généralement prononcés, puis translittérés en caractères italiques à leur première occurrence, selon le système défini par Turrell Wylie. 
certains aspects et entrainant la mise en relief de caractéristiques existantes en médecine tibétaine - ce qui revient souvent à créer de nouvelles significations. Ce double principe, combinant simultanément pertes et accentuations dans la traduction du savoir, procure aux interlocuteurs des praticiens des idées parfois erronées sur la médecine du Tibet.

Dans cette expérience culturelle, un dialogue s'instaure entre "vérités partielles » (Mumford, I989, p. II), rendant compte à la fois de la nature des échanges humains dans d'innombrables situations et, de façon plus spécifique dans le cadre de ce texte, des malentendus et des décalages conceptuels entre les praticiens et leurs patients. " Médecine », " science », " religion », « croyances », « énergies » sont, par exemple, autant de termes qui prennent des significations différentes d'un individu à l'autre, même si ces derniers s'entendent suffisamment pour avoir un semblant de dialogue.

On ne saurait cependant comprendre ces formes d'énonciation du savoir comme étant seulement dépendantes de la présence d'étrangers et du nouveau marché de la médecine tibétaine dans la région. Les processus de traduction du savoir médical tibétain ne peuvent pas davantage être réduits à des questions de langage ; ils prennent toute leur épaisseur et tout leur sens lorsque l'on examine aussi le contexte social général de production du discours et ses dimensions cognitives. Cet article montre que pour étudier quelques expressions dominantes du discours des amchi face aux patients internationaux - comme la « religion » et la « spiritualité tibétaine »-, il convient de considérer également l'architecture du savoir érudit en milieu tibétain et l'identité des amchi urbains dans le contexte sociopolitique et religieux du Ladakh.

\section{CADRE D'ÉTUDE}

Dans la région du Ladakh, la médecine tibétaine s'adresse principalement au marché local : la circulation mondiale des thérapeutes et l'export de médicaments sont des phénomènes récents et sociologiquement significatifs, mais ils demeurent limités à un faible nombre de praticiens. La dimension internationale de la médecine tibétaine est essentiellement relative à la clientèle des amchi urbains durant la période estivale. Depuis le milieu des années 1970 , le développement considérable du tourisme saisonnier draine dans son sillage une proportion aujourd'hui non négligeable d'étrangers provenant des pays industrialisés d'Occident et d'Asie, dont l'intérêt pour la médecine tibétaine relève plus de la découverte culturelle que de la quête de soins. La visite d'une clinique de médecine tibétaine fait généralement partie de l'itinéraire touristique des étrangers concernés. De fait, peu de « patients ॥ sont effectivement malades lorsqu'ils se rendent chez les praticiens. Ils consultent au mieux pour des affections qu'ils ne jugent pas comme potentiellement délétères, tels que les dérangements gastriques ou intestinaux passagers et les légers maux de tête 
dus à l'altitude (en cas de soupçons de maladie d'altitude et de l'apparition de symptômes plus inquiétants, ils se rendent préférentiellement à l'hôpital biomédical). La vaste majorité consulte dans l'objectif d'obtenir un bilan sur leur état de santé, de se faire "rééquilibrer » ou simplement par curiosité ou intérêt pour les médecines alternatives. L'image positive du Tibet dans nos contrées (Lopez, 1998) et l'engouement international pour les soins non biomédicaux contribuent à ce phénomène.

Cette configuration distingue mon étude des travaux réalisés sur les médecines asiatiques en Europe, où la démarche des consultants, qui peut également (mais pas seulement) se rapporter à une quête de bien-être ou de développement personnel, n'est pas systématiquement motivée par un désir de découverte. Au Royaume-Uni, les patients des cliniques du Tara Institute se dirigent vers la médecine tibétaine par choix thérapeutique, souvent par opposition idéologique à la biomédecine (perçue comme violente et déshumanisée) ou lorsque celle-ci n'a su leur apporter de réponse satisfaisante à leurs maux (au plan biologique ou en termes de relations humaines ou de cadres explicatifs du sens de leur mal) et souvent au fil d'un parcours thérapeutique diversifié (Millard, 2008). Certains patients deviennent assidus à la médecine tibétaine, ce qui n'est généralement pas le cas au Ladakh, où la majorité des consultants s'en remettent aux amchi pour la première fois. Nombre d'entre eux sont cependant familiers des médecines alternatives et du vocabulaire courant dans ce domaine, comme « énergie » et « holisme ». Ils ont tendance à faire un amalgame conceptuel entre des pratiques qui sont parfois très éloignées les unes des autres.

C'est dans ce contexte que s'est établi, avec une intensité soutenue depuis le milieu des années 1990, un nouveau marché médical tibétain au Ladakh. Les exilés tibétains ont été très réactifs afin de profiter de ce marché. L'édification du Men-Tsee-Khang Cultural Center de Leh, la capitale régionale, en 2000, a été partiellement envisagée en raison de l'augmentation saisonnière de la population internationale dans la région ${ }^{4}$.

The scope for a Tibetan Cultural Centre is vast because many western tourists visit Ladakh every year for $5 / 6$ months from April/May. (...) For 5 to 6 months in a year starting from April, Ladakh has heavy and increasing inflow of western tourists, many of whom have great interest in Tibetan medicine and astrology and other aspects of Tibetan Buddhism and Culture. (...) Ladakh attracts many tourists from different western countries, most of whom know and wish to know more about Tibet and Tibetans. (...) There are many Tibet related periodicals and magazines, both priced and unpriced in various languages, notably English, French,

4 Le centre de Leh a été édifié pour des raisons relatives au marché local et international des médecines traditionnelles : la présence de touristes et la production de médicaments. Le centre approvisionne la pharmacie centrale du Men-Tsee-Khang (sman rtsis khang, Collège de Médecine et d'Astrologie) de Dharamsala en matière médicale. L'institut de Dharamsala est le plus important consommateur de plantes himalayennes en Inde et le Ladakh est une zone de collecte privilégiée (Tenzin Choeudrak, cité par Tager, 1999). 


\begin{abstract}
German, Spanish etc. (...) According to the list provided by the Library of Tibetan Works and Archives, Dharamsala, there are nearly 200 Foreign Language periodicals and magazines related to Tibet and Tibetans. (...) A series of lectures, short term courses, sermons etc., especially relating to Tibetan medicine and astrology will be one of the important activities of the Centre, especially during the tourist season from April to September. (...) Many western tourists are now taking Tibetan medicine and a clinic in the Centre will be great benefit to the tourists, those using our library, those coming for the course/lectures etc. ([http://www.men-tsee-khang. org/aboutus/ladculture.htm], site Internet de l'Institution centrale à Dharamsala. Dernière consultation en décembre 2009).
\end{abstract}

Comme leurs homologues tibétains, quelques praticiens ladakhis aguerris à l'anglais et appartenant à l'élite de la médecine tibétaine urbaine ont également saisi cette occasion pour s'adresser directement aux étrangers par l'entremise de cliniques et d'associations dédiées à la préservation de leur médecine. Cette situation est indicative des remaniements à l'œuvre sur le marché de la santé au Ladakh. Sur les douze cliniques de médecine tibétaine de Leh, pendant la période de recherche entre 1998 et 2003 - mon présent ethnographique - , trois ont choisi une démarche promotionnelle agressive. Situées près des centres touristiques ou des endroits de passage, ces cliniques annoncent leurs services par des affiches illustrées en anglais que l'on trouve sur les murs des "German Bakeries », des " Cyber-Cafés », des agences de randonnée et dans tous lieux caractérisés par leur affluence en visiteurs étrangers. Certains se font connaître par l'intermédiaire de sites Internet en langue anglaise, française ou allemande. La visibilité de ces thérapeutes s'est trouvée remarquablement accrue et l'économie de leur clinique fonctionne désormais partiellement grâce à des ressources exogènes.

Ces cliniques font partie intégrante de programmes associatifs plus largement dédiés au « développement de la médecine des amchi ». Les praticiens concernés proposent des consultations, exposent les activités conduites par leurs associations et offrent divers types de services, de la vente d'eau potable à des "laveries écologiques ». Les organisations espèrent ainsi développer leur clientèle, promouvoir leur médecine et générer quelques fonds pour leurs activités. Des amchi organisent également dans ce cadre des conférences publiques d'introduction à la médecine tibétaine. Ces conférences sont tenues en anglais de façon régulière ou à la demande de quelques groupes organisés de voyageurs. Ces consultations et ces conférences ont fourni l'essentiel du matériel ethnographique réuni pour mon propos. Je retiens dans ce qui suit deux cas ethnographiques qui illustrent quelques logiques à l'œuvre dans les interactions entre amchi et patients étrangers. 


\section{UN NOUVEAU TERME PHYSIOPATHOLOGIQUE}

Une quinzaine de patients fréquentent chaque jour la clinique $Z$. Ils sont reçus dans une salle d'attente où se trouvent divers documents relatifs aux autres activités de l'organisation, impliquée dans la formation de jeunes amchi, l'établissement de cliniques villageoises et la conservation des plantes médicinales. Jessica s'est rendue sur place en août 2003 sur les conseils de son époux. Elle patiente une vingtaine de minutes, observant d'un air à la fois admirateur et interrogatif les multiples boîtes transparentes de pilules tibétaines qui tapissent les murs de la salle. Lorsque vient son tour, Jessica se présente à l'amchi comme étant une femme canadienne, « amoureuse de l'Himalaya ».

— [L'amchi] De quoi souffrez-vous $?^{5}$

— [Jessica] De rien en particulier, je voudrais obtenir un bilan énergétique.

L'amchi ne dit rien, mais invite Jessica d'un geste de la main à s'assoir près d'elle sur un petit tabouret. Tandis qu'elle remonte tour à tour les manches de sa patiente afin de préparer le diagnostic par la prise des pulsations radiales, elle l'interroge sur ses habitudes alimentaires. Jessica répond en s'indignant du fait que la nourriture est bien trop épicée en Inde. L'amchi sourit.

- Je vais prendre votre pouls en utilisant ces trois doigts [elle indique pour chacune de ses mains, l'index, le médius et l'annulaire]. Je les appose ainsi au niveau de l'artère radiale afin de diagnostiquer votre état de santé, au travers de l'état de vos organes. Ma main droite me permet par exemple de sentir [« to feel »] vos poumons, le gros intestin, le foie, le rein droit, la vessie et la rate.

Jessica écoute avec un intérêt manifeste. L'amchi se concentre sur son diagnostic et poursuit.

- Ma main gauche me renseigne sur six organes supplémentaires. À chacun de mes doigts correspondent deux organes, pour un total qui compte donc douze organes. Je lis ( «I read ») d'abord le pouls constitutionnel, qui m'informe sur vos énergies. Nous comptons trois énergies principales en médecine tibétaine, qui sont chacune subdivisées en cinq formes et possèdent des fonctions propres.

— Oui, je sais bien que mon énergie est très basse. Je peine à me déplacer.

L'amchi donnait au terme énergie un sens très différent de celui de sa patiente. Elle me confia que l'énergie avait pour elle et dans ce cadre le sens strict (mais complexe) de celui des nyespa (nyes pa) ${ }^{6}$. Il s'agit des principes physiologiques et

5 Les échanges entre amchi et patients étrangers rapportés dans cet article se sont tenus en langue anglaise. J'en donne ma propre traduction.

6 Les amchi donnent au terme énergie des sens évidemment multiples, qui recouvrent par exemple la force ou l'électricité. Utilisé dans le contexte de la consultation clinique, ce terme m'a toujours été rendu pour signifier les nyespa. 
pathologiques fondamentaux en médecine tibétaine, communément traduits dans la littérature spécialisée par humeurs ${ }^{7}$ et équivalents, dans leur état pathogène, aux doșa (" défaut, vice ») de l'āyurveda. L'énergie évoquée par l'amchi avait pour elle un sens très concret. Ce nouveau vocable signifiait un savoir théorique élaboré, qu'elle n'avait guère le temps d'expliquer, même sommairement, lors de ses consultations ${ }^{8}$. Elle précisa auprès de moi, en ladakhi, que «le terme énergie [elle le prononça en anglais] est ce que les étrangers comprennent le mieux lorsque je parle des nyespa. (...) Ils le connaissent et en parlent souvent ! Ce terme est plus compréhensible pour eux que "nyespa" ou même "humeurs" [qu'elle prononce également en anglais]. (...) II faut qu'ils aient une idée des principes fondamentaux. Utiliser des termes avec lesquels ils sont familiers facilite leur compréhension, ou du moins stimule leur intérêt. Pour eux, les nyespa ne sont pas palpables, alors que l'énergie est... [elle s'arrête un instant, dubitative], elle est imaginable. »

Elle ne se souciait toutefois guère de la polysémie du terme, qui, dans ce contexte, ouvrait en effet le champ à des interprétations très imaginatives. Les patients remarquaient justement cependant que l'« énergie » était au fondement de l'existence, elle était force de vie - un amchi dirait de même pour les nyespa. Pour Jessica comme pour un échantillon significatif de touristes interrogés suite aux consultations avec cet amchi, la médecine tibétaine se fondait sur une théorie des «flux énergétiques ». Les pulsations radiales étaient perçues lors du diagnostic comme des " énergies » qui transitaient par les organes et que l'amchi était présumé capable de décoder. Pour certains, l'amchi était sensible aux « radiations du corps »; il percevait l'« aura » de ses patients. Les étrangers employaient ce terme de façon assez variable mais toujours distincte de ce que le praticien signifiait exactement. Ces perceptions étaient paradoxalement confortées, alors que l'intention du praticien était de communiquer des idées claires à ses interlocuteurs.

Un récent ouvrage publié par un amchi ladakhi remarque par exemple :

« Le corps est constitué par de nombreuses forces invisibles, telles que des flux d'énergie, qui œuvrent constamment au fonctionnement du corps et de l'esprit. (...) Notre corps abrite un système complexe de canaux au travers desquels circulent divers types d'énergie, neurale, vasculaire ou lymphatique » (Phuntsog, 2006, p. 30).

Les amchi ladakhis ne sont évidemment pas les seuls à formuler ainsi les fondements physiopathologiques de leur médecine. Le terme est couramment utilisé dans les ouvrages en anglais produits par les Tibétains exilés en Inde (cf. Drungsto, 2004 et 2007 ; Men-Tsee-Khang, 200I). Il est également employé par les amchi en Europe et aux États-Unis. Tsering Gyatso, un praticien provenant du prestigieux Collège de Médecine et d'Astrologie (Mentsikhang) de Lhassa,

7 Lire Yonten Gyatso pour une critique de la traduction du terme nyespa (2005/2006).

8 Sur la théorie physiopathologique, voir Meyer (1995, 2006). 
remarqua par exemple en 2005 lors d'une conférence à l'université de Stanford, que la médecine tibétaine était une " médecine de l'énergie », c'est-à-dire une médecine reposant sur des " principes énergétiques » et ayant été établie dans un lieu, le Tibet, également doté d'une « énergie » favorable au développement spirituel :

« The medicine of energy is found in Tibet. Diseases emerge from the disequilibrium of the three main energies (...) The energy of the place itself in Tibet is a perfect setting to meditate and develop clarity of mind ॥ (Gyatso, 2005).

Ce nouveau terme (exogène et dépourvu d'équivalent exact dans la médecine tibétaine) peut être ainsi employé de façon assez variable selon les amchi - il est toujours en usage pour rendre les nyespa dans le contexte de la consultation clinique mais sa signification ne se limite pas à cela. Face à cette multiplicité, les connaissances des patients sont brouillées. Les praticiens tendent à formuler leur savoir médical de façon simple et à utiliser un nouveau vocabulaire pour en décrire les notions. Leur désir d'adaptation et de communication donne lieu à des interprétations parfois hasardeuses.

Aujourd'hui commun en médecine tibétaine, l'usage du terme énergie est très répandu dans le milieu du Nouvel Âge et des médecines alternatives en Occident (Berger, 2005 ; English-Lueck, 1990 ; Schmitz, 2006). Jean-Jacques Wunenburger remarque à ce propos : "Presque tous les adeptes des médecines alternatives ont intégré plus ou moins, dans leur compréhension de l'homme, la notion d'énergie. (...) On pourrait même penser que le recours privilégié, répétitif, à ce terme dans un discours signe l'appartenance au champ des médecines alternatives ॥ (2006, pp. 183-184). C'est probablement pour cette raison que certains observateurs (et les patients dans le contexte de cette étude) font l'amalgame entre toutes ces formes de médecines, des traditions très anciennement établies aux syncrétismes contemporains du Nouvel Âge, quelles que soient leur réalité et les significations effectives du discours des praticiens. Wunenburger lui-même passe sans explication de la notion d'énergie dans les médecines alternatives en Occident aux « flux énergétiques 》 dans la pensée et la médecine chinoises (ibid., p. 185). C'est ainsi que la médecine tibétaine se retrouve parfois incluse dans la « nébuleuse mystique-ésotérique 》 qui unifie, selon Françoise Champion (1994), les religiosités parallèles et certaines formes thérapeutiques hétérodoxes. Pourtant, le simple usage du terme énergie n'autorise pas cette inclusion sans en étudier son sens dans un contexte donné.

Les cliniques du Tara Institute au Royaume-Uni évitent ce problème par l'adoption d'une autre terminologie et une insistance sur son explication auprès des patients. Colin Millard souligne l'usage inhabituel du terme énergie lors des consultations. Lorsque tel est le cas, le terme signifie vitalité (2008). L'auteur poursuit : "The three humours were not described as three 'energies' in the body; rather they where described as groups of psychological and physiological 
processes. Because they consist of material, dynamic and psychological components, at the Tara Institute they are referred to as 'bio-dynamic agents' » (ibid., p. 204). Ces agents bio-dynamiques sont expliqués aux patients, lesquels disposent également d'un petit ouvrage d'introduction à la médecine tibétaine qu'ils ont la liberté d'étudier. Au fil des consultations, les patients sont d'une certaine manière éduqués par leurs praticiens, qui ont une expérience clinique quotidienne auprès de patients non tibétains et une meilleure connaissance de leurs attentes et de l'environnement général, très compétitif et régulé, des médecines alternatives dans le pays. Les amchi tibétains exploitent une niche et déploient les moyens appropriés pour être compris et fréquentés. Selon Millard, la question de l'inintelligibilité par les patients du discours des praticiens est hors de propos dans le contexte de son étude.

Dans ce cas, les agents bio-dynamiques signifient également les principes physiologiques et pathologiques humoraux, c'est-à-dire les « énergies » au sens où l'amchi du Ladakh les conçoit. En comparaison, la clientèle étrangère des amchi ladakhis est saisonnière et relativement faible en proportion sur leur nombre total de consultations. Ces nouveaux patients sont bienvenus mais les efforts déployés pour les attirer ou les éduquer au savoir médical tibétain sont bien moins formellement organisés.

Au Ladakh, l'adoption du terme énergie rend compte d'une forme de mimétisme discursif qui ne signifie pas que les praticiens s'approprient pour autant de nouveaux concepts ou de nouvelles notions ${ }^{9}$. Lorsque j'ai évoqué cette possibilité au cours d'une discussion en anglais avec la consultante de Jessica, celle-ci rétorqua : «Depuis près de dix ans, je pratique la médecine que m'a transmise mon professeur ${ }^{10}$. Je l'enseigne aujourd'hui conformément à nos textes [elle fait référence à des textes médicaux pluriséculaires] et mes enseignements se déroulent en tibétain ! [elle n'a donc pas besoin de recourir à la traduction] J'apprends bien sûr beaucoup par ma pratique clinique, mais je m'instruis de façon identique auprès des gens d'ici et d'ailleurs. (...) » Elle poursuit, espiègle : « J'apprends beaucoup des patients étrangers, oui. J'apprends à connaître leur vie et à parler anglais ! Je me suis aussi fait quelques amis. ॥

Pour elle, l'utilisation d'un nouveau terme avait peu à voir avec la façon dont elle se formulait son savoir. Cette amchi utilise un terme récurrent dans le discours des étrangers pour décrire sommairement les notions physiopathologiques de sa médecine : le mot énergie sert à décrire des conceptions préexistantes et déjà bien établies. Aucune de mes données ethnographiques n'autorise à dire que sa pratique et son savoir ont été significativement transformés depuis qu'elle est régulièrement exposée à des patients étrangers. Elle communique

9 Pour une étude sur les implications du mimétisme dans le domaine médical, lire Langford (2002), et plus généralement dans le domaine social, Adams (1996) et Taussig (1993).

10 Cette amchi est issue d'une formation institutionnelle, accessible sur dossier après l'équivalent du baccalauréat, dans laquelle elle a étudié la médecine tibétaine pendant six années. Son enseignant se rapproche ainsi plutôt d'un professeur d'université que d'un maître comme on le trouve parfois dans les formations lignagères. 
de façon pragmatique en s'efforçant de présenter son savoir dans un langage et d'une manière compréhensibles.

Les résultats de cette démarche en termes de communication demeurent cependant rarement convaincants. La simplification de connaissances très complexes et la faible durée des interactions ne permettent généralement pas aux patients de bien comprendre le savoir que souhaitent véhiculer les amchi et encore moins les fondements théoriques de leur médecine. Les praticiens sont contraints à sélectionner des éléments de leur savoir, qui apparaissent dès lors comme relevant d'une importance particulière. Cette manière de procéder occulte de nombreux autres aspects de la médecine tibétaine qui ne sont pourtant pas moins centraux.

\section{LE DÉROULEMENT DU DIALOGUE}

L'amchi porte une attention maximale aux pulsations de Jessica. Elle se concentre, comme si elle éprouvait des difficultés à effectuer son diagnostic. Elle semble écouter les pulsations. La praticienne fait la moue puis fixe intensément Jessica, interrogative : « Je ne trouve pas votre rate ».

Jessica est abasourdie. Elle marque un long silence stupéfait, puis confie à l'amchi qu'elle souffrait d'une grave affection de la rate qui a conduit à une ablation de l'organe. Elle se tourne vers moi en s'exclamant : " l'amchi est aussi douée de pouvoirs divinatoires ! ». La praticienne explique qu'elle s'est contentée de mettre en pratique le savoir médical théorique qui lui a été transmis". Pour Jessica, l'amchi « sait plus qu'elle ne veut bien le reconnaître ». Elle estime qu'elle est douée d'une forme de vision intérieure (« inner vision ») acquise par des années de méditation bouddhiste. Ce type de conclusion est régulièrement avancé par les patients, sans que leur condition et le résultat du diagnostic ne soient aussi extraordinaires que dans le cas de Jessica. La curiosité qui conduit les étrangers à fréquenter ces cliniques laisse alors place à la stupéfaction lorsqu'ils considèrent comme juste le diagnostic prononcé par leur praticien. Les modèles explicatifs alors mobilisés invoquent souvent, sous des formes discursives certes variées, la divination et le sacré, à défaut de pouvoir expliquer les procédures diagnostiques en leurs propres termes ${ }^{12}$.

II La pratique n'est cependant jamais l'exacte projection de la théorie. Le savoir théorique présente par exemple diverses étapes dans le diagnostic. Le praticien est censé faire appel à trois méthodes d'investigation : I) l'inspection (aspect et couleur de la peau, de la langue et de l'urine en particulier, pour laquelle l'aspect, l'odeur et le goût sont examinés), 2) l'interrogation (facteurs causals, localisation de la maladie, symptomatologie) et 3) la palpation (température du corps, anormalités physiques et prise des pouls radiaux). La prise des pouls, qui est certes l'aspect le plus important du diagnostic, est en pratique le seul examen sérieusement effectué avec l'inspection de la langue. Pour une analyse détaillée de la sphygmologie tibétaine, voir Meyer (1981 et 1992). Le cas des cliniques du Tara Institute contrastent de nouveau avec cette situation. Millard note que l'examen d'urine et l'inspection de la peau y sont couramment pratiquées (2008, p. 197).

12 Selon la théorie médicale, l'examen des pouls et de l'urine peut toutefois être effectué à des 
Certains détails du diagnostic n'ont pas échappé à Jessica. Elle reformule sa pensée :

- [Jessica] Je vous ai vu fixer avec insistance le Bouddha peint sur cette toile [il s'agit d'un thangka ${ }^{13}$ ]. Je pense que vos pouvoirs proviennent de là. C'est son propre pouvoir thérapeutique que vous canalisez (« you must channel the Buddha's healing power »).

- [L'amchi, amusée] Lorsque le diagnostic n'est pas aisément établi, je me concentre sur Sangyé Smanla (sangs rgyas sman bla), oui. Je lui demande de m'aider, c'est tout. Notre médecine provient de ce Bouddha. C'est lui qui détient le savoir originel. (...) Ce savoir est transmis de génération en génération depuis plus de deux mille ans.

Poser le regard sur Sangye Smanla en espérant bénéficier de son aide pour arriver à identifier correctement la maladie n'est pas une pratique réservée aux étrangers. J'ai eu l'occasion de l'observer à maintes reprises dans les consultations villageoises (Pordié, 2007a, p. 106). Les amchi du Ladakh estiment que le savoir médical tibétain a été initialement délivré par Sangyé Smanla, le Bouddha Maître des Remèdes, une émanation du Bouddha historique Śākyamuni. Les praticiens du Ladakh attribuent au Bouddha le contenu du texte canonique de référence de la médecine tibétaine, le Gyud-zhi (rGyud-bzhi) ou Quadruple Traité, dont l'histoire et les origines demeurent obscures. Sa version actuelle semble avoir été élaborée au Tibet aux alentours du XII ${ }^{e}$ siècle, malgré la présence fort probable de pratiques médicales érudites dans ce pays depuis la seconde moitié du VIIe siècle. Ce texte a été ensuite complété et développé, notamment lors d'un travail de révision et de réécriture orchestré au XVIIe siècle par Sangyé Gyamtso (I653-1705), le régent du $V^{e}$ Dalai Lama (Meyer, 1995). L'origine supposément divine de cet ouvrage et les faits historiques qui lui ont donné naissance sont généralement conciliés par les amchi qui considèrent ce texte comme un terma (gter ma), c'est-à-dire comme un texte caché puis redécouvert, selon une tradition indotibétaine (Mayer, 1994). La période de « redécouverte » du terma correspond généralement à la période historique de compilation du texte en question, qui est ensuite rétrospectivement attribué à des Maîtres religieux du passé. Le texte se retrouve ainsi pourvu d'une plus grande ancienneté, d'une autorité peu discutable et d'un caractère sacré. Ainsi, les praticiens donnent au Gyud-zhi le statut de " parole de Bouddha ». Ce savoir se serait ensuite transmis de proche en proche. Les praticiens remarquent que la connaissance de la médecine ne suffit pas à elle seule pour être totalement initié. Les amchi doivent pour cela recevoir un enseignement tantrique, lors de cérémonies dénommées wang lung

fins divinatoires, comme pour l'identification des esprits maléfiques (Meyer, 1995, p. 135). Mais ces techniques aujourd'hui désuètes n'ont jamais été pratiquées lors des nombreuses consultations que j’ai pu observer au cours des douze dernières années.

I3 Les thangka (thang kha) sont des toiles peintes, généralement suspendues et encadrées de beaux tissus, sur lesquelles sont habituellement représentées des divinités. II existe aussi une série de peintures médicales, regroupées en quatre-vingt thang kha et créées au XVIIe siècle, illustrant le savoir médical théorique tibétain. Voir le travail fondamental de Parfionovitch et al. (1992). 
(dbang lung), dont le sens recouvre les notions d'acquisition de pouvoir et de transmission par la lecture (Pordié, 2007a). Le Bouddha, comme l'a perçu Jessica, tient un rôle très central dans la pratique des amchi.

- [l'amchi] Je lui [Sangyé Smanla] accorde toute ma dévotion. Mais notre médecine est une science, c'est une science bouddhiste...

La médecine tibétaine est une " science traditionnelle » (rig gnas) (voir la section suivante sur l'architecture du savoir) et c'est à cela que l'amchi faisait référence. Elle pensait préciser de cette façon le caractère érudit, logique et cohérent de son savoir complexe. Les praticiens distinguent tout à fait la " science des soins " (sowa rigpa, gso ba rig pa) de la science " moderne » (tshan rig, un néologisme), même si dans certaines situations une ambigüité peut-être maintenue - Vincanne Adams expose ce cas particulier au Tibet où l'usage rhétorique de la science par les amchi, passant de rig gnas à tshan rig, permet de séculariser (et donc de dépolitiser) la médecine tibétaine dans le contexte politique chinois (200I). Lorsqu'ils soulignent le caractère scientifique de leur médecine ${ }^{14}$, les amchi du Ladakh différencient généralement leur science de la science moderne. Au Ladakh, le Dr. S. T. Phuntsog écrivit ainsi que si la « science des amchi » apparaissait discutable pour les biomédecins, c'est qu'ils en ignoraient le contenu : " In the name of civilization and modernisation, some of the traditional systems of Indian medicine are considered as superstitious and dogmatic by the allopathic practitioners. The truth lies in the ignorance about Amchi science in those people »(Phunstog, 1995: 34). L'association principale d'amchi défendit de la même manière l'un de ses projets auprès de la Commission Européenne en soulignant que la médecine tibétaine « était l'un des plus anciens systèmes dans l'histoire des sciences médicales » (LAS, n.d. [200I ?]). Les amchi de cette région ne remettent généralement pas en question la légitimité scientifique de leur pratique. Mais sans autre explication sur les frontières qui distinguent une science et l'autre, le malentendu est favorisé. En introduisant la science, un autre terme pourvu de significations variables selon les parties en présence, l'amchi introduisit plus de confusion encore.

Elle s'adresse alors en Ladakhi à son assistante en lui demandant de réunir deux types de pilules, puis lance à Jessica qu'elle n'avait jamais traité de patient dépourvu de rate. Elle l'assure du fait que les médicaments lui seront utiles. Elle lui conseille aussi de suivre un régime adéquat pour les sept jours suivants, puis de revenir consulter.

Au déjeuner, Jessica remarque auprès de sa famille que cette amchi est « exceptionnelle ».

— [Jessica] Elle [l'amchi] a vu pour ma rate. Est-ce que vous imaginez ? Elle a juste pris mon pouls. Elle m'a dit qu'elle pratiquait une science bouddhiste. Ces gens méditent et deviennent clairvoyants. (...) C’est une médecine énergétique.

I4 Le caractère scientifique de la médecine est également revendiqué dans d'autres cas en Inde, comme en médecine yūnānī (Liebeskind, 2002) ou ayurvédique (Cohen, 1995). 
Qualifiée dans aucun des domaines évoqués par la praticienne, la patiente reconstruit le discours de l'amchi de façon erronée. Elle ne modifie ni ses préconceptions sur les fondements énergétiques de la pratique ni ses idées sur sa dimension divinatoire. Bien au contraire, elle m'apprend que ces qualités de clairvoyance proviennent d'une science (de la divination), en l'occurrence bouddhiste.

- [Le mari] C'est incroyable, je te l'avais dit. [II s'adresse alors à moi] Hier, l'amchi a diagnostiqué mon ulcère. Elle m'a dit que j'avais un problème à l'estomac, des douleurs après la prise des repas.

- [Jessica] Leurs connaissances proviennent du Bouddha, te rends-tu compte? Plus de deux mille ans... Ce savoir dépasse notre propre entendement. II est à mi chemin entre la médecine, la religion et la magie. Ils évaluent nos énergies et remettent tout en ordre !

L'interaction clinique entre Jessica et sa praticienne dura moins de dix minutes. L'amchi s'efforça d'abord d'expliquer simplement quelques fondamentaux du diagnostic par la prise des pulsations radiales. Les deux interlocutrices se sont ensuite entendues sur le terme énergie, qui pourtant désignait pour l'une des principes physiopathologiques relevant d'un savoir théorique et pour l'autre une conception très abstraite du corps et la raison de son état de fatigue. Les interrogations de la patiente déplacèrent enfin le dialogue vers les relations entre médecine et bouddhisme. Le déroulement de la consultation, retransmise ici en quasi intégralité, se poursuivit de façon fragmentée, en abordant très superficiellement un ensemble de domaines considérablement complexes et sujets à des interprétations variées. Finalement, la discussion véhicula une version distordue du savoir thérapeutique tibétain et de la pratique clinique. Jessica quitta la clinique avec des idées erronées sur la pratique médicale dont elle venait de faire l'expérience.

Venue au Ladakh à la recherche de l'altérité et d'un Orient éloigné, la patiente fut satisfaite de son interaction avec la praticienne.

Lorsque, je fis part à l'amchi de cet échange, celle-ci remarqua qu'il était habituel pour elle d'être considérée comme pratiquant quelque forme de divination bouddhiste car ses patients étaient ignorants des fondements théoriques de son savoir clinique. ${ }^{15}$ II convient de préciser que ces mêmes patients sont, bien entendu, également ignorants de la théorie biomédicale - mis à part pour les quelques biomédecins qu'il m'a été donné de rencontrer dans les cliniques tibétaines. En revanche, les pratiques communes de leur médecin de famille ou la médecine prédictive de pointe ne leur apparaissent généralement pas comme de la divination. Ne pas comprendre le langage ou les idées de leur médecin est

I5 Tsewang Dolkar Khangkar, femme amchi issue d'une lignée tibétaine très réputée, remarque également à propos de sa pratique à New Delhi : « [Les patients] me regardent comme si je détenais des pouvoirs magiques, comme si je possédais une énergie secrète me différentiant des autres créatures. Moi qui ne suis ni sage ni érudite, encore moins philosophe, il m'arrive souvent de les détromper » (Khangkar et Lamothe, $1997:$ II). 
envisageable sans chercher à expliquer leurs modes opératoires dans le registre magique ou divinatoire car son savoir repose sur la science (biomédicale). C'est une chose acceptée, considérée comme un domaine de spécialistes et conférant à ces derniers une certaine autorité. C'est cette même croyance partagée en la science qui a pour effet de disqualifier (ou de reléguer au rang du magique) « celui qui tentent de faire exister des pratiques différentes, s'adressant autrement au malade » (Stengers $1997: 55$ ), comme dans le cas des amchi.

L'amchi poursuivit : « Je ne peux ni les éduquer ni leur parler en termes plus simples. L'important est qu'ils prennent leurs médicaments ! S'ils estiment que notre médecine est efficace, ils développeront un intérêt qui à son tour les incitera à découvrir l'étendue de la « science des soins » (sowa rigpa). Pour ceux qui le désirent, nous organisons chaque vendredi une conférence d'introduction à la médecine tibétaine. »

Je connaissais bien ces conférences puisque j'y ai assisté de très nombreuses fois pendant plusieurs étés. Mais je n'étais pas persuadé pour autant qu'elles suffisent à établir un espace de compréhension réciproque entre praticiens et étrangers. Passons donc maintenant au second cas ethnographique : les exposés publics illustrent également la façon dont le dialogue peut-être orienté vers la religion.

Un amchi présente les fondamentaux de sa médecine à un groupe de touristes nord-américains lors d'un après-midi de la fin du mois de juillet 2002. Le responsable du groupe introduit l'exposé de son invité en résumant ce qui pour lui sont les principes fondateurs de la médecine tibétaine. L'échange se tient bien sûr en anglais. Il explique : « la philosophie [de la médecine tibétaine] est de traiter les maladies sans perturber le fonctionnement des organes, défini comme un flux d'énergie circulant le long de points discernables qui se dénomment chakras. (...) Les potions et les onguents, ainsi que d'autres choses, comme les shampoings et les herbes, sont appliqués sur le corps dans des circonstances mystiques et souvent magiques ". L'amchi écouta poliment, sans relever les absurdités formulées par cet homme. Les Ladakhis évitent soigneusement les désaccords publics (Pirie, 2007). II me confie plus tard, amusé, qu'il ne fait rien de magique lorsqu'il délivre un traitement et ne produit ni ne procure de shampoings. Le fonctionnement des organes n'est pas davantage défini comme l'affirme l'américain.

Face à ces propos, l'amchi ne manifeste pas son désagrément. Il entame son exposé en abordant les diverses formes de traitements et leur chronologie : Iles recommandations relatives au régime alimentaire, 2- les recommandations relatives aux comportements ; 3 - les médicaments à base de plantes, dont certains incluent des pierres semi-précieuses et des métaux, et 4- les thérapies externes. II détaille chacun de ces points pendant une quinzaine de minutes puis ajoute enfin, " il y a également des pratiques méditatives bouddhistes. ॥ II fait référence à des formes de traitements possiblement donnés à des patients affectés par des dérèglements profonds du vent organique (loung nyespa, 
rlung nyes pa) qui conduisent à des troubles mentaux. II ne précise pas que ces traitements échappent à la fois au domaine de compétences de la médecine et des amchi. Kim Gutschow le souligne dans son étude sur la détresse mentale au Zangskar, en montrant que c'est parce que la maladie mentale signifie à la fois des problèmes d'ordre physiologiques et sociaux, que l'amchi ne peut à lui seul permettre la guérison de son patient (sous presse). Son domaine d'application du savoir est, en effet, strictement médical ; il ne peut remplacer des recours spécifiques d'ordre religieux, que les patients effectuent dans ce cas auprès de moines bouddhistes ou d'oracles.

À l'écoute de la remarque de l'amchi sur la méditation bouddhiste, le responsable américain coupe net son interlocuteur, pensant préciser sa pensée. II déclame sur un ton assuré un ensemble d'idées fausses : « ce sont des pratiques tibétaines uniques, comme l'imposition de mains, les cristaux correspondant aux chakras énergétiques. L'énergie est le Qi, elle circule dans le corps. ॥ II conclut que la médecine tibétaine est « très populaire chez les occidentaux cherchant dans la magie un antidote aux poisons de la médecine scientifique », ne réalisant pas à quel point il en reproduisait les discours. ${ }^{16}$

L'amchi ne fait aucun commentaire. Son silence est perçu comme un consentement. II tente ensuite d'expliquer la pratique complexe du palper des pouls radiaux : " la médecine occidentale se base sur la prise de la pression sanguine et sur le son du flux sanguin perçu par l'intermédiaire du stéthoscope, qui renseigne aussi sur l'état du cœur. (...) Les amchi perçoivent de nombreuses différences lors de la prise du pouls. La précision diagnostique dépend aussi de la pratique bouddhiste du praticien, de ses initiations tantriques ». Le responsable du groupe nord-américain remarque que cette perspective est certainement la plus attrayante pour les charlatans occidentaux, amateurs de légendes relatives aux pouvoirs tantriques. L'amchi, quant à lui, soulignait ainsi l'imbrication entre deux domaines du savoir érudit. Pour lui, la « science des soins » et la pratique du bouddhisme se combinent pour leur bénéfice mutuel.

L'amchi ne concevait pas la médecine tibétaine comme étant l'apanage des praticiens tantriques. Dans sa description très élémentaire du savoir médical tibétain figuraient aussi des références au bouddhisme. Bien que ces références ne fussent absolument pas dominantes dans ses propos initiaux, leur seule présence dans un discours sur la médecine suffit à les distinguer. Le responsable du groupe américain, manifestement intéressé par ces aspects de la pratique, saisi chacune de ces occasions pour réorienter la discussion.

I6 Cette situation est assez commune. Michael Cohen, professeur à Harvard et spécialiste des "médecines complémentaires », souligne la nécessité de comprendre les méthodes nonbiomédicales dans leurs propres termes, dont le « mystérieux diagnostic par la prise du pouls » en médecine tibétaine: «Those terms of reference may include framing healing as a transfer of consciousness, healing intention, or therapeutic information through the medium of "spiritual energy" - for lack of better terminology - that is, through as yet unknown mechanisms » (2007 : 2). II ne remet pas en cause l'efficacité thérapeutique de la médecine tibétaine, mais succombe à une idée répandue : les mécanismes d'action de la médecine tibétaine reposent sur des principes inconnus. Voir également à ce titre, les remarques de Jobst sur cette médecine (1998). 
Avant de continuer plus loin dans la retranscription de cet échange, intéressons nous brièvement aux relations entre médecine et bouddhisme dans le savoir tibétain savant.

\section{L'ARCHITECTURE DU SAVOIR}

Les amchi affirment qu'un bon thérapeute est d'abord un bon technicien, dont la connaissance de la théorie médicale est sans faille mais ils ajoutent avec force que la réalisation de rituels bouddhistes et le progrès sur la voie du dharma développe les qualités individuelles de chacun, la prise en charge des patients et la thérapeutique. Les amchi, comme la population générale du Ladakh, sont caractérisés par une forte religiosité et ils ne conçoivent par conséquent pas leur exercice médical sans recourir régulièrement à la religion. Ainsi, le recours à des techniques bouddhistes, comme lors des cérémonies de consécration des médicaments, vient occasionnellement ponctuer l'exercice médical. La récitation de textes religieux, la prière, la méditation, l'invocation de divinités interviennent régulièrement dans la vie des praticiens afin d'améliorer leur pratique médicale ou de potentialiser leurs médicaments (Pordié, 2007a et 2008a). Le discours de l'amchi lors de l'exposé auprès du groupe nord-américain rendait non seulement compte d'une expérience pratique qui pour lui était effective, mais aussi d'une conception érudite des relations entre médecine et bouddhisme inscrite dans l'architecture du savoir scholastique tibétain.

Les principes de la doctrine bouddhique ont joué un rôle important dans la construction de cette médecine savante ${ }^{17}$, en fondant notamment l'éthique médicale et quelques aspects de la théorie, comme les causes primordiales et la classification des maladies (Meyer, 1988). Selon Vincanne Adams, « at the level of theory, morality is what ties all causal factors together. (...) The closer one gets to the theoretical explanations of Tibetan diagnoses, the closer one gets to fundamentally religious ideas that explain such things as the relationship between perception and physical process which is mediated by morality » (200 I, pp. 236237). "La théorie médicale, concernée par un aspect du malheur humain, devint une application particulière de la doctrine bouddhique de la libération. Les mêmes processus psychologiques et moraux qui enchaînent les êtres au monde phénoménal sont donc à l'origine de la vie organique et à l'œuvre dans la survenue des désordres inhérents à sa nature "(Meyer 1987, p. 237). Un remarquable effort de synthèse entrepris par les érudits tibétains et étalé sur plusieurs siècles a donné naissance à un art médical original ayant le bouddhisme de tradition Mahāyāna comme cadre religieux de référence.

17 La médecine tibétaine est issue de sources multiples, dont l'āyurveda et la médecine de Chine ont fourni les apports principaux. 
La tradition bouddhique tibétaine a formulé la place de la « science des soins » dans le cadre des sciences scholastiques. Ces sciences érudites (rig gnas, skt. vidyā sthāna) sont composées de cinq domaines de connaissance majeurs (rig gnas che ba Inga) et de cinq domaines mineurs (rig gnas chung ba Inga). La médecine (sowa rigpa) appartient aux rig gnas majeurs, au même titre que les arts et les techniques, la linguistique/grammaire, la logique/épistémologie et la " science intérieure » (nang gi rig gnas). Parmi ces cinq domaines, seule « la science intérieure » est spécifique, " non commune » (thun mong ma yin pa'i rig gnas), au bouddhisme. Les quatre autres sont des domaines de connaissance communs (thun mong rig gnas) au bouddhisme et à d'autres traditions telles que le brahmanisme. S'ils sont en ce sens différenciés du bouddhisme, ces domaines communs n'en sont donc pas pour autant isolés. Ils relèvent du chos (dharma) ${ }^{18}$, dont le champ sémantique est plus vaste que celui de religion.

La médecine peut ainsi entrer dans le cadre du chos selon les finalités qui lui sont assignées ou selon le cadre intentionnel dans lequel elle s'inscrit. C'est ainsi lorsque la médecine est considérée comme nécessaire au progrès sur la voie du dharma. Dans le monde tibétain, la médecine entre de cette manière dans le champ d'action du bouddhisme lorsqu'elle est mise au service d'un projet de nature religieuse ${ }^{19}$. En revanche, même lorsque le cadre intentionnel est considéré comme favorable aux progrès sur la voie, la médecine appartient à des plans de réalité ou de vérité qui n'ont de pertinence que jusqu'à une certaine étape de la voie. Les sources de références de la médecine ont été ainsi intégrées au canon bouddhique, tout en distinguant celle-ci, en tant que science dite " extérieure », des enseignements considérés comme proprement bouddhiques, la science « intérieure ». L'enseignement monastique peut, par exemple, intégrer l'étude de textes médicaux. Dans ce cas, l'objectif est l'apprentissage de l'une des sciences majeures du canon bouddhique et non nécessairement la pratique de la médecine. À l'inverse, le savoir des amchi, bien que spécialisé à la médecine, peut tout à fait intégrer des conceptions élaborées sur la « science intérieure ». Tandis que le chos est perçu par les praticiens comme relevant des sciences « extérieures » et « intérieures » et la médecine comme non réductible à son domaine de validité/vérité, le savoir médical demeure différencié (mais non isolé) du savoir religieux. Selon Janet Gyatso, une « mentalité médicale » distincte du bouddhisme a été forgée au Tibet dès le $\mathrm{XVl}$ e siècle $(2004)^{20}$. La constitution d'une épistémè propre à la médecine, à cette époque et lors du siècle suivant, rend particulièrement compte d'un désir d'autonomie éprouvé par les médecins, d'une certaine appréciation de la matérialité et d'un sens de la vérité empirique.

I8 Le dharma correspond aux enseignements du Bouddha. II enseigne l'essence douloureuse de toute chose et la loi naturelle qui les régit.

19 Frances Garrett le montre bien dans une étude conduite sur les textes médicaux constituant l'anthologie tibétaine des Dix-huit pratiques additionnelles (Cha lag bco brgyad) - un corpus datant du milieu du XII ${ }^{\mathrm{e}}$ siècle au milieu du XIII ${ }^{\mathrm{e}}$ siècle (2006).

20 C'est-à-dire environ un siècle avant la centralisation de l'État tibétain, la création des premières institutions d'enseignement médical (1643) - dans lesquelles se trouvaient essentiellement des moines -, et la réécriture du Gyu-zhi, l'ouvrage canonique dont il fut question plus haut. 
Comme c'est le cas de la quasi-totalité des pratiques et des savoirs tibétains érudits, la médecine se déploie sur des registres différents. Leur savoir chevauche divers domaines de connaissances. Ce qui caractérise l'interaction des amchi ladakhis avec les étrangers ne relève pas de la transformation de la pratique médicale mais de la mise en avant d'un pan de leur savoir de manière sélective. Les discours de l'amchi lors de son exposé étaient aussi inscrits dans une tradition savante. Ce savoir vaut d'ailleurs à ces praticiens d'être considérés par leurs concitoyens comme étant des « érudits » (khaspa, mkhas pa).

La complexité de la tradition scholastique tibétaine échappe à de nombreux amchi, y compris ceux de l'élite médicale urbaine dont il est question ici. En revanche, ils en connaissent parfaitement les grandes lignes, telles que je les ai exposées jusqu'ici dans cette section. C'est d'ailleurs auprès de deux d'entre eux, dont l'amchi concerné ici, que j'ai moi-même acquis ces connaissances, avant de les confronter avec celles de leurs homologues puis avec des sources textuelles secondaires. II s'agit donc également de matériaux ethnographiques. Cette remarque est importante, car elle met en perspective la suite de la conversation entre l'amchi et le groupe américain que nous allons découvrir maintenant.

Comprenant mal la remarque du responsable du groupe à propos du tantrisme et des charlatans occidentaux - il ne connaissait pas le terme utilisé en anglais (« conman ») et pensait que le responsable du groupe l'encourageait à poursuivre sa présentation en notant les imbrications avec le bouddhisme -, il continue son exposé en évoquant brièvement les nyespa. II insiste donc délibérément sur certaines caractéristiques relevant expressément du bouddhisme.

- [L'amchi] La médecine tibétaine est une médecine humorale, comme la médecine ayurvédique ou la médecine grecque antique, d'où provient la médecine moderne. (...) II existe trois énergies principales, que nous appelons nyespa (...). Ces trois nyes pa dépendent des trois poisons mentaux du bouddhisme [dug gsum, les obscurcissements primordiaux de l'esprit] : le vent provient du désir, la bile de la haine [" hatred » qui correspond à zhe sdang, aversion] et le phlegme de l'obscurité mentale. (...) La cause lointaine des maladies est l'Ignorance ( $m a$ rig $p a$ ), que nous pouvons définir comme étant une perception erronée du monde phénoménal ${ }^{2 !}$. Les causes premières [de la maladie] sont les nyes pa. La phase pathologique est déclenchée par des facteurs favorables liés au mode de vie, à la diététique, aux variations saisonnières, aux répercussions des mauvaises actions antérieures, à un traitement erroné, aux poisons et aux esprits maléfiques.

2 I L'Ignorance est d'abord la cause de l'existence biologique puisqu'elle produit les trois humeurs par l'intermédiaire des trois poisons mentaux. Ce n'est que secondairement, et de manière donc inhérente à l'existence biologique, qu'elle est aussi l'origine lointaine des maladies. 
- [L’Américain] Vous parlez des esprits maléfiques. Quel est donc le rôle de la croyance dans la médecine amchi, je veux dire au sens de la croyance religieuse ?22

- Il est nécessaire de croire en l'amchi et au bouddhisme pour que le système fonctionne pleinement. Les modifications comportementales sont, par exemple, guidées par des principes bouddhistes, comme les perfections [" perfections », phar phyin] à mettre en œuvre pour celui qui s'engage sur la voie du bodhisattva ${ }^{23}$.

- Je vous suis. En fait, votre médecine relève plutôt de la religion que de la santé publique!

C'est sur cette remarque que l'exposé s'est conclu. De nombreuses équivoques et décalages importants entre des conceptions comme " bouddhisme », " religieux » ou " croyances » sont apparus dans cet extrait. L'orientation de la discussion autour des dimensions religieuses de la médecine et du rôle de la doctrine bouddhiste dans les conceptions médicales fit ressortir un ensemble de termes qui n'ont pas le même sens pour chacun des interlocuteurs. Le responsable du groupe était manifestement surpris par la continuité que la science médicale tibétaine entretient avec d'autres domaines du savoir, notamment religieux, qui déstabilise, en effet, la rationalité d'ordre naturaliste dont rend compte la théorie médicale. C'est pourtant cette continuité qui permet aux amchi de déplacer leurs propos d'un domaine du savoir à l'autre sans y percevoir d'incohérence.

\section{LA PRODUCTION SOCIALE DU DISCOURS}

L'amchi n'a pas su éclairer correctement ses interlocuteurs. II s'efforça d'expliquer dans un temps très réduit ce qu'était la médecine tibétaine et retint pour cela des éléments qu'il a jugés " compréhensibles et intéressants " pour son audience. Pour en comprendre les raisons, il faut s'intéresser à l'histoire économique et politique de la région. Depuis 1947, date à laquelle le Ladakh fut annexé à l'État du Jammu et Cachemire, l'espace politique régional est animé par une série de demandes officielles ayant pour objectif l'autonomie administrative. Ces mouvements autonomistes ont parfois conduit à des manifestations très violentes, comme en 1989, mais ils n'ont cependant jamais été dirigés contre I'Union Indienne. Ils expriment un radicalisme religieux refusant l'administration

22 II est intéressant de noter ici que la théorie physiopathologique médicale n'est pas en mesure de rendre compte de l'action pathogène des mauvais esprits (Meyer, 2006, p. 17).

23 Le bodhisattva (Être promis à l'éveil) renonce au nirvāna - ou extinction, qui marque la fin de l'existence cyclique dans le saṃsāra - afin de contribuer à ce que l'ensemble des êtres sensibles l'obtienne à son tour. L'idéal bouddhique du bodhisattva est un sujet récurrent dans les textes médicaux qui alimentèrent de nombreux débats entre savants dans le Tibet pré-moderne (Schaeffer, 2003). 
du Ladakh par le Cachemire musulman. Ces agitations conduites par les autorités bouddhistes ladakhies ont permis l'obtention d'une autonomie partielle en 1995, sans parvenir à l'apaisement du climat social. Bien au contraire, les populations, composées en parts à peu près égales de musulmans (non cachemiris) et de bouddhistes, ont été fragmentées et les différences religieuses au sein même du Ladakh ont été exacerbées ${ }^{24}$.

Dans ce contexte, l'industrie du tourisme qui génère une proportion très significative de l'économie régionale, a participé au réagencement des relations interethniques et des identités religieuses. Le tourisme a tenu un rôle déterminant dans la mise en exergue de la culture bouddhiste, dont l'un des aspects les plus représentatifs est la médecine savante locale. Le tourisme " ethnique » et " culturel » prévaut depuis 1974, date à laquelle cette région fut ouverte aux étrangers ${ }^{25}$. L'industrie touristique assure la promotion de l'ethnicité et de la culture bouddhiste au Ladakh, entendus au sens de produits commerciaux à proposer aux visiteurs nationaux et internationaux (Michaud, 1990 et 1993). Certaines caractéristiques sont ainsi mises en relief, comme le folklore, la médecine ou l'environnement monastique, pendant que d'autres demeurent sous silence. Cette démarche est nourrie et consolidée par les représentations positives et souvent romantiques que les touristes ont du Ladakh ${ }^{26}$. Cette région apparaît comme un Éden bouddhiste épargné de l'oppression sévère dont a souffert le Tibet lors de la Révolution culturelle chinoise. Aux yeux des visiteurs, le Ladakh est l'un des rares lieux préservés du bouddhisme tibétain. Alex Gillespie estime que ces interactions entre touristes et Ladakhis ont partiellement produit la « culture ladakhie » (2006) ${ }^{27}$. L'Islam, pourtant présent dans la région depuis le $\mathrm{XIV}^{\mathrm{e}}$ siècle, n'est généralement pas considéré comme faisant partie intégrante du pays ${ }^{28}$.

Le tourisme "culturel » est ainsi sélectif. Ce parti pris semble d'ailleurs paraître à certains auteurs comme allant de soi. James Crowden avance par exemple que « far from destroying the culture they [les touristes] have I think given a greater awareness to the local people of the worth of their culture and in particular the worth of their monasteries and the paintings and artefacts associated with them » (Crowden, 1997, p. 60). Soulignant ainsi maladroitement

24 Lire au sujet des relations entre musulmans et bouddhistes, les travaux de Aggarwal (2004), Gutschow (2006) et van Beek (200I, 2004).

25 Certaines zones n'ont été cependant accessibles que près de vingt ans après pour des raisons de défense (Roaf, 1995). Le nombre de touristes s'est accru de 3000 pour cent entre 1974 et I997, avec une moyenne supérieure à 15000 visiteurs annuels depuis le début des années 1980 (Singh Jina, 1997). Le tourisme n'a pas ensuite cessé d'augmenter, sauf en 1999 et 2000 en raison du conflit de Kargil.

26 Voir, plus généralement, Lopez (1998) et Brauen (2004) au sujet des représentations des Occidentaux sur le monde tibétain.

27 Ailleurs, au sujet de l'identité sherpa et du tourisme, lire l'ouvrage d'Adams (1996).

28 Comme l'a montré Siddiq Wahid (1989), l'idée que les Ladakhis musulmans sont étrangers dans leur propre pays est également longtemps restée dominante dans les travaux universitaires. Patrick Kaplanian distinguait par exemple en termes peu élogieux les Baltis (musulmans) des Ladakhis " proprement dits », c'est-à-dire, selon lui, des communautés bouddhistes (198I). On retrouve aussi ce portrait des musulmans ladakhis dans certains récits de voyage (cf. Harvey, 1983). 
le caractère ethnicisant du tourisme (Picard et Wood, 1997), cet auteur reprend malheureusement à son compte les politiques partisanes du gouvernement ladakhi.

Les dynamiques politiques régionales et l'établissement d'un contact de plus en plus étroit entre les Ladakhis et les touristes sont des éléments fondamentaux qui expliquent l'émergence du sentiment identitaire contemporain au Ladakh. Cette situation façonne les idées que les amchi ont d'eux-mêmes et des attentes des touristes à leur égard. Ainsi, l'amchi chargé d'exposer sa médecine au groupe nord-américain remarqua plus tard auprès de moi, en anglais :

"Je suis conscient du fait qu'une forte proportion de touristes occidentaux est sympathisante ou intéressée par la découverte du bouddhisme. Ils viennent par milliers chaque été pour visiter nos monastères. Certains étudient même le bouddhisme. (...) Lorsque sa sainteté [le Dalai Lama] vient au Ladakh les étrangers sont présents de façon massive. (...) En soulignant quelques aspects du bouddhisme auprès des Américains l'an passé [nous sommes en 2003], je pensais répondre à leur demande, comprendre leur intérêt. J'ai développé certaines choses en répondant à leurs questions mais je n'ai rien dit au fond [il s'esclaffe à cette idée]. Notre savoir est vaste. (...) Ils ont eu l'air satisfaits, n'est-ce-pas ? L'important est qu'ils apprennent un peu et repartent heureux. Je ne pouvais pas tout leur dire, ni même corriger leurs idées erronées. Notre médecine est très différente de ce qu'ils connaissent et leur chef [« boss », c'est-à-dire le responsable du groupe] était très sûr de lui ! (...) Nous avons parlé du bouddhisme, selon leur souhait. (...) J'aurais pu insister sur les aspects scientifiques de la médecine ou relever l'existence de pharmacopées et de formulaires médicaux très détaillés, mais ce n'était pas utile face à ce type d'auditoire. Il en est autrement, ajouta-t-il, lorsque je m'adresse aux autorités nationales en charge de la recherche clinique sur les médecines traditionnelles !»

L'amchi pensait donner une image valorisante de sa pratique médicale en relevant quelques aspects relatifs au bouddhisme. Ce sentiment est partagé par d'autres praticiens du Ladakh, qui ont tendance à souligner les aspects les plus éthérés de leur pratique lorsqu'ils s'adressent aux touristes étrangers. La brochure en anglais de l'organisation principale d'amchi ladakhis signale ainsi : " The definition of 'health', according to this system, is not just freedom from disease but implies mental and spiritual elevation, in addition to the concept of physical 'healthiness'. The fundamental concept of this system relates to the essential unity of human nature - the inseparability of mind, body and spirit. The treatment of physical ailments through somatic methods is thus combined with the treatment of mental problems through meditation, incantation and moral development » (brochure de présentation diffusée en 2002). La quatrième de couverture de la brochure de Ladakh Society for Traditional Medicines entend résumer en termes identiques le contenu de son propos sur la médecine tibétaine : «Western biomedicine focuses primarily on the physical body; Sowa 
Rigpa (the Tibetan science of healing) concerns the body, mind and soul » (LSTM, n.d. [2006?]).

II faut, pour comprendre cette démarche, faire appel au concept d'« identité médicale caméléon ॥ (Pordié, 2008b, pp. 148-152). L'identité médicale des amchi est certes homogène dans un contexte donné et auprès d'interlocuteurs spécifiques mais elle prend des allures différentes de contexte en contexte. Par exemple, ces praticiens mettent en avant leur parenté avec l'āyurveda auprès des autorités nationales en charge des médecines traditionnelles afin d'être d'abord compris pour ensuite mieux établir leur différence. De même, lorsqu'ils s'adressent à des biomédecins ou, comme l'amchi le dit lui-même, à des équipes de recherche médicale, leur discours est construit autour d'une logique naturaliste, relevant ainsi certains aspects de la théorie, l'usage de plantes médicinales et leur potentiel biologique. Un principe similaire est notable avec les étrangers : ils ne modulent pas seulement leur discours pour établir leur singularité mais également dans l'intention de rendre leur médecine accessible et attractive. L'identité médicale des amchi ne se limite pas à ce seul aspect, elle le contient.

Le désir de communication de l'amchi et les personnes en présence ont conduit à mettre en relief le rôle du bouddhisme dans la pratique médicale. Le praticien a relevé certains caractères de sa médecine de façon sélective afin de communiquer avec ses interlocuteurs étrangers. Ces accentuations occultent une partie significative de leur savoir, notamment relatif à la théorie, à la pratique clinique ou à la préparation des remèdes. Comme l'indique Deborah Roots dans un contexte pourtant très différent, ce principe véhicule des versions fragmentaires, « désarticulées » (1996, p. 93), du savoir médical tibétain qui empêchent l'établissement d'un vrai dialogue entre les parties concernées.

\section{CONCLUSION}

Les flux de personnes, d'objets et d'idées qui caractérisent les déplacements transnationaux entre divers endroits du monde rendent possibles l'émergence de savoirs hybrides ou syncrétiques, tout autant qu'ils façonnent les désirs individuels marqués par la recherche d'identités « authentiques » (Appadurai, 1996). Des formes de soin inspirées des médecines ayurvédique et chinoise connaissent par exemple des logiques très similaires, se transformant assez profondément au contact des populations d'Occident. Mêlant adroitement commerce et spiritualité, ces nouvelles pratiques s'inscrivent désormais dans la nébuleuse du milieu New Age ${ }^{29}$. Dans le registre thérapeutique, le New Age

29 II convient de préciser que le Tibet est une composante fondamentale de l'idéologie New Age : « [Tibet's] close geographic proximity with India and its philosophical connection with Buddhism 
se caractérise par des pratiques syncrétiques, qui s'apparentent à de nouvelles formes de religiosités et qui allient divers savoirs thérapeutiques et diverses techniques de soin, dont les paradigmes sont parfois très éloignés (Beckford, 1984 ; English-Lueck, 1990 ; Wunenburger, 2006 : 145-I52). Les praticiens qui enseignent ces médecines grossissent parfois les traits relatifs aux domaines religieux et spirituels afin de positionner leur tradition sur le marché en visant particulièrement les étudiants en quête de transformation personnelle. La médecine chinoise en Amérique du Nord est à ce titre particulièrement évocatrice. Paul Unschuld remarque que ces pratiques de marketing véhiculent une image distordue de ce savoir thérapeutique, présenté comme holistique et opposé à la biomédecine (2009). Certaines formes mondialisées de l'āyurveda sont également unifiées par " la réinterprétation ou la création d'aspects spirituels et philosophiques » (Wujastik et Smith, 2008, pp. 2-3). De nouvelles formes thérapeutiques apparentées à la tradition ayurvédique se déclinent ainsi en variantes multiples, reposant sur des réinterprétations et des réinventions plurielles. Jean Langford a spécifiquement examiné ces transformations et leurs significations sociales dans le cadre des recours thérapeutiques transnationaux en Inde (2002). Elle montre que les praticiens ayurvédiques intègrent les discours, les concepts et les fantaisies d'une clientèle cosmopolite autant séduite par les médecines alternatives que par « la culture et la spiritualité indienne ». Ces changements résultent de logiques mimétiques et d'appropriations diverses, qui conduisent à des bouleversements épistémologiques et à des modifications profondes de la pratique. Sur le marché de la santé en Amérique du Nord, écrit Sita Reddy (2002), ces transformations permettent de combler l'absence de reconnaissance officielle et institutionnelle de l'āyurveda. Dénommées « New Age Ayurveda » (Zysk, 200I), ces variantes sont parfois observées avec condescendance. Elles sont dévalorisées par rapport à la Grande Tradition dont on regrette la dilution et l'acculturation au profit de pratiques hybrides à forte portée commerciale. Ce type d'écueil doit être absolument évité. C'est la généalogie des pratiques de soin, comme l'a excellemment démontré Francis Zimmermann (1995), qui importe.

Geoffrey Samuel s'est intéressé à la médecine tibétaine dans un contexte similaire à celui présenté dans cet article : cette médecine en Inde - chez les Tibétains exilés dans son cas - y est également destinée à des patients internationaux. II note que les « perspectives spirituelles » de la médecine tibétaine sont rendues explicites dans les cliniques destinées aux touristes par le déploiement d'objets religieux, l'ambiance recueillie et l'atmosphère quasi monastique (200I, repris par Janes, 2002). Les praticiens participeraient à un phénomène ainsi rendu global. Mais l'enchantement auquel cet auteur fait référence ne serait-il pas une apparence trompeuse ? Une apparence « qui nous tromperait tous par le seul fait que nous prenons pour "enchantement" ce qui

placed it at the center of the New Age imagination, yet its misperceived isolation conveniently served to preserve the aura of distance and mystery needed for the location of inaccessible spiritual masters and esoteric teaching 》 (Korom, 200I, p. I8I). 
le serait pour nous si on nous l'offrait, alors que cela ne l'est pas nécessairement pour ceux dont il est le quotidien » (Benoist, 2002, p. 476). L'observation des cliniques destinées aux touristes dans la région du Ladakh conduit aux mêmes conclusions que le travail de Samuel. Mais il en va presque exactement de même pour toutes les "cliniques" villageoises (qui sont généralement des pièces domestiques), ainsi que pour les bureaux gouvernementaux dédiés à la médecine des amchi. Les unes et les autres sont pourvus d'images et de photographies religieuses, de statues, de bâtonnets d'encens et de thang kha figurant aussi bien des planches anatomiques que le Bouddha Maître des Remèdes. Les Ladakhis ne considèrent d'ailleurs pas les cliniques «pour touristes » très différentes des autres - excepté, peut-être, en ce qui concerne les étalages de médicaments mieux achalandés et mieux organisés, ou le niveau plus élevé et la fixité des tarifs. Un touriste sensible à une « ambiance spirituelle », éprouve ce sentiment dans n'importe quelle clinique, et ce d'autant plus, remarquons le au passage, quand elle est tenue par un moine-amchi, ce qui correspond au cas exposé par Samuel (200I, p. 262) ${ }^{30}$.

En outre, les changements semblent profonds chez les exilés tibétains. Samuel remarque que l'établissement de la médecine tibétaine " s'est accompagné d'interactions avec des étrangers en quête de sagesse médicale tibétaine qui ont entrainé sa reconstitution pour les consommateurs occidentaux » (Samuel, 200 I, p. 262). Le marché déterminerait in fine le sort de la pratique médicale : la médecine tibétaine est produite et transformée afin de satisfaire aux désirs des patients internationaux. Cet auteur ajoute qu'en raison « de la popularité du bouddhisme tibétain sur la scène spirituelle internationale, il n'est pas surprenant de constater que l'image d'une approche holistique de la santé fondée sur une approche spirituelle de la vie soit devenue dominante [en médecine tibétaine] » (200I, p. 262). Ce type de logique est incontestablement vérifié, notamment en Occident (cf. Janes 2002, pp. 282-284 ; Vargas, 2008), mais il se combine à d'autres dynamiques sociales. Tandis que le processus de sécularisation de la médecine tibétaine en exil, observé par Fernand Meyer il y a près de vingt ans (1992a, p. 2), semble, en effet, s'atténuer de nos jours, il semble par exemple que le « ré-enchantement » de la médecine tibétaine au Men-Tsee-Khang de Dharamsala soit orienté par les politiques tibétaines nationalistes et le contenu des curricula médicaux (Prost, sous presse). Ces formations intègrent des rudiments de biomédecine et insistent parallèlement sur le rôle et l'importance du bouddhisme pour la pratique médicale. L'appropriation fragmentaire de la science et l'accentuation de la religion semblent caractériser aujourd'hui l'institution médicale tibétaine dominante chez les Tibétains exilés en Inde (ibid.). Cette situation pourrait également avoir une incidence sur les discours tenus dans les cliniques que Samuel a observées.

De mon point de vue, il ne s'agit pas de l'expression d'un processus global

30 Samuel retient comme exemple la clinique de Yeshi Dhonden à Dharamsala, qui est à maints égards exceptionnelle. Yeshi Dhonden est un moine, ex-amchi du Dalai Lama, sa réputation est internationale et une foule de touristes se masse chaque jour devant sa clinique. 
d'enchantement et de transformation de la médecine tibétaine destinée aux patients internationaux, mais d'une accentuation pragmatique du savoir, qui peut-être dictée par des raisons très diverses, comme le désir d'adaptation à la clientèle éprouvé par certains praticiens ou les politiques nationalistes des exilés tibétains. Cet article a permis d'identifier quelques logiques à l'œuvre au Ladakh dans la formulation en langue anglaise des principes physiologiques et pathologiques fondamentaux en médecine tibétaine (les nyespa/energies) et dans la mise en relief de références au religieux, à la croyance et au bouddhisme dans le discours des amchi. Les conceptions des personnes en présence en matière de médecine, du rapport au corps et à l'esprit, du « religieux » ou de rationalité ont certes une complexité et une diversité qui demandent assurément un approfondissement. Cette étude permet toutefois d'émettre quelques conclusions : l'appropriation de nouveaux termes et la mise en avant du « religieux » et du bouddhisme est la conséquence des efforts entrepris par les amchi pour dialoguer avec des étrangers habituellement intéressés par les aspects de la médecine fondés sur la « religion » et la « spiritualité ». Elle devient compréhensible par l'étude conjuguée du contexte sociopolitique régional contemporain et du savoir scholastique en milieu tibétain. Le bouddhisme tient une importance particulière dans la vie des praticiens, la religion caractérise l'identité des praticiens au Ladakh et son articulation avec le savoir médical est inscrite dans une tradition érudite très ancienne.

Les modifications observables dans le discours des praticiens n'indiquent aucun signe de transformation des pratiques cliniques ou du savoir théorique, mais correspondent à un registre « intermédiaire » permettant une communication, qui relève d'ailleurs du malentendu. Les relations entre médecine et religion sont probablement influencées par le nouveau marché local et les amchi reproduisent dans une certaine mesure les discours de leurs patients internationaux, mais rien ne permet de dire qu'ils en intègrent aussi les idées. II s'agit d'un mimétisme discursif qui ne permet pas davantage de conclure de façon téléologique sur la reconstitution de la médecine pour le marché.

La mise en évidence du " religieux » en médecine tibétaine peut tromper l'observateur. Les changements sociaux, qui caractérisent l'environnement de la médecine tibétaine au Ladakh comme ailleurs, ne sont pas toujours consubstantiels à des modifications profondes des savoirs et des pratiques (Pordié, 2008c, pp. 17-19). Les transformations discursives et les accentuations pragmatiques étudiées dans cet article ne sont pas (encore ?) conjuguées à des transformations profondes dans les domaines de la cognition et de l'épistémologie médicales. En étudiant ainsi le savoir médical tibétain, ce texte a plus généralement interrogé la production du savoir anthropologique sur les médecines d'Asie dans des contextes d'exercice médical renouvelés, comme c'est le cas avec les patients étrangers. Il convient de déjouer l'illusion d'optique juste évoquée et de se prémunir contre une illusion d'option qui souscrit sans toujours la nuancer à la théorie de la grande transformation des médecines traditionnelles aujourd'hui. 


\section{RÉFÉRENCES}

Adams, V. (2002). Randomized controlled crime: postcolonial sciences in alternative medicine research, Social Studies of Science 3, 32(5): 659-690.

Adams, V. (200I). The Sacred in the Scientific: Ambiguous Practices of Science in Tibetan Medicine, Cultural Anthropology 16(4), 542-575.

Adams, V. (1996). Tigers of the Snow and Other Virtual Sherpas. An Ethnography of Himalayan Encounters, Princeton: Princeton University Press.

Aggarwal, R. (2004), Beyond Lines of Control: Performance and Politics on the Disputed Borders of Ladakh, India, Durham and London: Duke University Press.

Appadurai, A. (1996). Modernity at Large: Cultural Dimensions of Globalization, Minneapolis: University of Minnesota Press.

Aschoff, J. C., Tashigang, T. Y., Maier, J. (1999). Clinical trial in migraine prophylaxis with a multicomponent Tibetan jewell pill. Transfer problems of Tibetan into Western medicine, demonstrated "pars pro toto" on the Aconite plants in our Tibetan prescription, in J. C. Aschoff et I. Rösing (eds.). Tibetan Medicine. "East meets West - West meets East" (Proceedings of the International Symposium, University of Ulm, 19-20 July 1996), Ulm: Fabri Verlag.

Beckford, J. A. (1984). Holistic imagery and ethics in the new religious and healing movements, Social Compass 3I (2-3): 259-272.

Benoist, J. (2002). La maladie entre nature et mystère, in R. Massé et J. Benoist (eds.), Convocations thérapeutiques du sacré, Paris: Karthala.

Berger, C. (2005). Héros de la guérison. Thérapies alternatives aux États-Unis, Paris: Les Empêcheurs de Penser en Rond / Le Seuil.

Brauen, M. (2004). Dreamworld Tibet, Western Illusions, Trumbull, CT: Weatherhill.

Champion, F. (1994). La « nébuleuse mystique-ésotérique »: une décomposition du religieux entre humanisme revisité, magique, psychologique, in J.-B. Martin et F. Lapalantine (dir.), Le défi magique. Esotérisme, occultisme, spiritisme, Lyon: Presses Universitaires de Lyon.

Cohen, L. (1995). The epistemological carnival: Meditations on disciplinary intentionality and Ayurveda, in D. Bates (ed.), Knowledge and the Scholarly Medical Traditions, Cambridge: Cambridge University Press.

Cohen, M. (2007). Healing at the Borderland of Medicine and Religion, New Delhi: Orient Longman.

Craig, S. (2006). On the 'Science of Healing'. Efficacy and the Metamorphosis of Tibetan Medicine, Ph.D. Dissertation, Cornell University, New York.

Craig, S., Adams, V. (2008). Global Pharma in the Land of Snows. Tibetan medicines, SARS, and identity politics across nations, Asian Medicine 4(I): I-28.

Crowden, J. (1997). The road to Padum: Its effect on Zangskar, in $\mathrm{H}$. Osmaston and N. Tsering (eds.), Recent Research in Ladakh 6, New Delhi: Motilal Banarsidass Publishers [first edition, Bristol, 1996].

Drungtso, T. T. (2007). Basic Concepts of Tibetan medicine. A Guide to Understanding Tibetan Medical Science, Dharamsala: Drungsto Publications.

Drungtso, T. T. (2004). Tibetan Medicine. The Healing Science of Tibet, Dharamsala: Drungsto Publications.

English-Lueck, J. A. (1990). Health in the New Age. A study in California Holistic Practices, Albuquerque: University of New Mexico Press.

Garrett, F. (2006), Buddhism and the historicizing of medicine in thirteenth-century Tibet, Asian Medicine 2(2): 204-224.

Gillespie, A. (2006). Becoming Other. From Social Interaction to Self-Reflection: Greenwich, Connecticut: Information Age Publishing. 
Gutschow, K. (sous presse). The interplay of amchi medicine and ritual treatments in Zangskar: A case of wind disorder, in L. Pordié (ed.), Healing at the Periphery. Ethnographies of Tibetan Medicine in India, London \& Durham: Duke University Press.

Gutschow, K. (2006). The Politics of Being Buddhist in Zangskar: Partition and Today, India Review 5(3-4): 470-498.

Gyatso, J. (2004). The authority of empiricism and the empiricism of authority: medicine and Buddhism in Tibet on the eve of modernity, Comparative Studies of South Asia, Africa and the Middle East 24(2): 83-96.

Gyatso, T. (2005). Introduction to Tibetan medicine: diagnosis, diet, \& behavior advice, Paper presented at the Stanford Medical School, Stanford University, 27 October.

Gyatso, Y. (2005/2006). Nyes pa: A brief review of its English translation, Tibet Journal 30(4)-3I(I): III I-I20.

Harvey, A. (1983). A Journey in Ladakh, London: Cape.

Janes, C. R. (2002). Buddhism, science, and market: The globalisation of Tibetan medicine, Anthropology \& Medicine 9(3): 267-289.

Janes, C. R. (200I). Tibetan medicine at the crossroads : radical modernity and the social organisation of traditional medicine in the Tibet Autonomous Region, China, in L. H. Connor and G. Samuel (eds.), Healing Powers and Modernity. Traditional Medicine, Shamanism and Science in Asian Societies, Westport, Connecticut - London: Bergin \& Garvey.

Janes, C. R. (1999). The health transition, global modernity and the crisis of traditional medicine: the Tibetan case, Social Science \& Medicine 48: I803-I820.

Janes, C. R., Hilliard, C. (2008). Inventing tradition: Tibetan medicine in the post-socialist contexts of China and Mongolia, in L. Pordié (ed.), Tibetan Medicine in the Contemporary World. Global Politics of Medical Knowledge and Practice, London \& New York: Routledge.

Janes, C. R., Chuluundorj, O., Hilliard, C., Rak, K., Janchiv, K. (2005). Poor medicine for poor people? Assessing the impact of neoliberal reform in a postsocialist context, Global Public Health I: 5-30.

Jobst, K. A. (1998). So what if there is no immediate explanation?, The Journal of Alternative and Complementary Medicine 4(4): 355-357.

Kaplanian, P. (198I), Les Ladakhis du Cachemire, Paris: Hachette.

Khangkar, D., Lamothe, M.-J. (1997). Médecin du toit du monde, Paris: Éditions du Rocher.

Kloos, S. (2006). The institutionalization of Tibetan medicine in the Indian exile, communication donnée lors des Séminaires en Sciences Sociales de l'Institut Français de Pondichéry, Pondichéry, 30 mai.

Korom, F. J. (200I). The role of Tibet in the New Age movement, in T. Dodin and $\mathrm{H}$. Räther (eds.). Imagining Tibet. Peceptions, Projections and Fantaisies, Boston: Wisdom Publications.

Langford, J. (2002). Fluent Bodies. Ayurvedic Remedies for Postcolonial Imbalance, Durham, NC. : Duke University Press.

LAS (n. d. [200I ?]). Project: To establish a fulfledge Amchi/Astro institute in Leh, [Proposal for the European Commission], Ladakh Amchi Sabha.

Liebeskind, C. (2002). Arguing science. Unani tibb, hakims and biomedicine in India, 1900-50, in E. Waltraud (ed.), Plural medicine, Tradition and Modernity, I800-2000, London and New York: Routledge.

Lopez, D. (1998). Prisoners of Shangri-la: Tibetan Buddhism and the West, Chicago: University of Chicago Press.

LSTM (n.d. [2006 ?]). Introduction. Amchi Medicine, The Ladakh Society for Traditional Medicines, Leh.

Mayer, R. (1994). Scriptural Revelation in India and Tibet, Tibetan Studies 2: 533-544.

Men-Tsee-Khang (200I). Fundamentals of Tibetan Medicine, Dharmasala : Men-TseeKhang.

Meyer, F. (2006). La rationalité théorique de la médecine tibétaine au prisme de son 
traité de référence : de la physiologie à la pharmacopée, in J. Fleurentin et J.-P. Nicolas (eds.), La médecines tibétaine. Sources, concepts et pratique actuelle (Actes de la journée du 8 avril 2006 à Metz), Metz: Société Française d'Ethnopharmacologie - Institut Européen d'Écologie.

Meyer, F. (1995). Theory and practice of Tibetan Medicine, in J. V. Alphen and A. Aris (eds.), Oriental Medicine. An Illustrated Guide to the Asian Arts of Healing, Serindia Publications (re-edited by Shambala Publ., Boston, 1997).

Meyer, F. (1992a). La démarche diagnostique en médecine tibétaine, in D. Gourevitch (ed.), Maladie et maladies. Histoire et conceptualisation, Genève: Droz.

Meyer, F. (1992b). Introduction: The Medical Paintings of Tibet, in Y. Parfionovitch, G. Dorje, and F. Meyer (eds.), Tibetan Medical Paintings: Illustrations to the Blue Beryl treatise of Sangye Gyamtso (I 653-I 705), 2 vol., New York: Harry N. Abrams, Inc.

Meyer, F. (1988). Médecine et bouddhisme au Tibet, Grand Atlas des Religions, Encyclopedia Universalis.

Meyer, F. (1987), Essai d'analyse schématique d'un système médical, la médecine savante du Tibet, in A. Retel-Laurentin (ed.), Etiologie et perception de la maladie, Paris: L'Harmattan.

Meyer, F. (I98I). Le système canalaire et la sphygmologie en médecine tibétaine d'après le Rgyud-bzhi et son commentaire, le Vaidūurya sngon-po, in Les médecines traditionnelles de I'Asie. Actes du Colloque de Paris (I I-I 2 juin 1979); Strasbourg: Université Louis Pasteur.

Michaud, J. (1993). Tourism as catalyst of economic and political change: the case of highland minorities in Ladakh (India) and Northern Thailand, Internationales Asienforum 24(I-2): $2 I-43$.

Michaud, J. (1990). Mais entrez donc! Les entrepreneurs touristiques et le pouvoir au Ladakh (Inde). Anthropologie et Sociétés I4(3): I27-139.

Millard, C. (2008). The integration of Tibetan medicine in the United Kingdom: The clinics of the Tara Institute of Medicine, in L. Pordié (ed.), Tibetan Medicine in the Contemporary World. Global Politics of Medical Knowledge and Practice, London and New York: Routledge. Mumford, S.T. (1989). Himalayan Dialogue. Tibetan Lamas an Gurung Shamans in Nepal, Madison: The University of Wisconsin Press.

Phuntsog, S. T. (2006). Ancient Metria [sic!] Medica. Sowa Riga (Tibetan Science of Healing), New Delhi: Paljor Publications.

Phuntsog, S.T. (1995). Amchi: Past \& Present. Adapted from the speech given by Dr. S.T. Phuntsog at the Amchi Conference in Leh, October 1994, Ladags Melong I(I): 34.

Picard, R., Wood, R. E., eds. (1997). Tourism, Ethnicity, and the State in Asian and Pacific Societies, Honolulu: University of Hawaii Press.

Pirie, F. (2007). Peace and Conflict in Ladakh: The Construction of a Fragile Web of Order, Leiden: Brill.

Pordié, L. (2008a). Reformulating ingredients: outlines of a contemporary ritual for the consecration of medicines in Ladakh, in M. van Beek and F. Pirie (eds.), Modern Ladakh. Anthropological Perspectives on Continuity and Change, Leiden and Boston: Brill.

Pordié, L. (2008b), Hijacking intellectual property rights. Identities and social power in the Himalayas, in L. Pordié (ed.), Tibetan Medicine in the Contemporary World. Global Politics of Medical Knowledge and Practice, London and New York: Routledge.

Pordié, L. (2008c). Tibetan medicine today. Neo-traditionalism as an analytical lens and a political tool, in L. Pordié (ed.), Tibetan Medicine in the Contemporary World. Global Politics of Medical Knowledge and Practice, London and New York: Routledge.

Pordié, L. (2007a). Buddhism in the everyday medical practice of the Ladakhi amchi, Indian Anthropologist 37(1): 93-116.

Pordié, L. (2007b). Médecine traditionnelle et conflits interreligieux au Ladakh, La Revue de l'Inde 7: 157-170.

Prost, A. (sous presse). Walking the middle way: Men-Tsee-Khang physicians in transition, in L. Pordié (ed.), Healing at the Periphery. Ethnographies of Tibetan Medicine in 
India, Durham, NC: Duke University Press.

Reddy, S. (2002). Asian medicine in America: the Ayurvedic case, The ANNALS of the American Academy of Political and Social Science 583(I): 97-I2I.

Rinpoche, S. (1998). The spiritual heart of Tibetan medicine, paper presented at The First International Congress for Tibetan Medicine, Washington DC, November.

Rinpoche, S. (1992). The Tibetan Book of Living and Dying, New York: HarperCollins Publishers.

Roaf, R. (1995). Ladakh in 1936, in H. Osmaston and P. Denwood (eds.), Recent Research on Ladakh, Vol. 4 \& 5, Delhi: Motilal Barnasidass Publishers.

Samuel, G. (200I). Tibetan Medicine in Contemporary India, Theory and Practice. in L. H. Connor, G. Samuel (eds.), Healing Powers and Modernity, Traditional Medicine, Shamanism and Science in Asian Societies, Westport, Connecticut - London: Bergin \& Garvey.

Schaeffer, K. R. (2003). Textual scholarship, medical tradition, and Mahāyāna Buddhist ideals in Tibet, Journal of Indian Philosophy 31:62 I-64I.

Schmitz, O. (2006). Soigner par l'invisible. Enquête sur les guérisseurs aujourd'hui, Paris: Imago.

Singh Jina, P. (1997). Impact of Tourism on the Ecology of the Ladakh Himalayas, in H. Osmaston and N. Tsering (eds.), Recent Research in Ladakh 6, New Delhi: Motilal Barnasidass Publisher.

Stengers, I. (1997). Sciences et pouvoirs. Faut-il en avoir peur ? Bruxelles: Labor.

Sulek, E. (2005). Mongolian doctors and Tibetan medicine in Poland, Paper given at the Workshop "Transcultural Interface and Local Implementations of Asian and Western Medical Systems: Transfer, Integration and Transformation between Asia and Europe", Central Asian Seminar, Institute for Asian and African Studies, Humboldt-University, February II- 12.

Tager, D. K. (1999). Tendzin Tcheudrak vu de l'intérieur, L'actualité des religions, $n^{\circ} 3$.

Taussig, M. (1993). Mimesis and Alterity. A Particular History of the Senses, New York and London: Routledge.

Thinley, P. (1997). Tibetan Medicine: Too successful for its own good?, Tibetan Review, Feb.: I4-19.

Unschuld, P. U. (2009). What is medicine?, Western and Eastern Approaches to Healing, Berkeley: University of California Press.

Vargas, I. (2008). Tibetan medicine revisited in the West. Notes on the integrative efforts and transformative consequences occurring in Massachusetts, USA, in L. Pordié (ed.), Tibetan Medicine in the Contemporary World. Global Politics of Medical Knowledge and Practice, London and New York: Routledge.

Van Beek, M. (200I). Beyond Identity Fetishism: 'Communal' Conflict in Ladakh and the Limits of Autonomy, Cultural Anthropology I5 (4): 525-569.

Van Beek, M. (2004). Dangerous Liaisons: Hindu Nationalism and Buddhist Radicalism in Ladakh, in S. Limaye, M. Malik, R. Wirsing (eds.), Religious Radicalism and Security in South Asia, Honolulu: Asia-Pacific Center for Security Studies.

Wahid, S. (1989), Riots in Ladakh and the genesis of a tragedy, Himal Sept.-Oct.: 24-25.

Wujastik, D., Smith, F. (2008). Introduction, in D. Wujastik and F. Smith (eds.), Modern and Global Ayurveda. Pluralism and Paradigms, New York: State University of New York Press.

Wunenburger, J.-J. (2006). Imaginaires et rationalité des médecines alternatives, Paris: Les Belles Lettres.

Zimmermann, F. (1995). Généalogie des médecines douces. De l'Inde à l'occident, Paris: Presses Universitaires de France.

Zysk, K. G. (200I). New Age Ayurveda or what happens to Indian medicine when it comes to America, Traditional South Asian Medicine 6: 10-26. 
Laurent PORDIÉ est anthropologue, chercheur au Pôle d'Excellence de l'Université de Heidelberg.

$\begin{array}{ll}\text { Adresse : } & \text { Karl Jasper Center } \\ & \text { Cluster of Excellence ‘Asia \& Europe' } \\ & \text { University of Heidelberg } \\ & \text { Voßstrasse 2, Gebaüde } 4400 \\ & \text { D-69l I5 Heidelberg } \\ & \text { Allemagne } \\ & \text { pordie@asia-europe.uni-heidelberg.de }\end{array}$

\section{Abstract: Accentuations and pragmatism. Tibetan MEDICAL KNOWLEDGE FOR FOREIGNERS}

Since the mid-1970s, the development of tourism in Ladakh, a remote region in the Indian Himalayas, has attracted an increasing number of foreigners interested in Tibetan medicine. Some English-speaking practitioners have taken the opportunity to directly address this new clientele, in their own clinics or through public lectures. This article is concerned with this local manifestation of therapeutic globalization. More specifically, it examines how these practitioners present an elaborated and complex body of medical knowledge with the intention of making it accessible to foreigners. The practitioners pragmatically attempt to create a space for communication, but neither their medical knowledge nor their practice are deeply altered for all that. The brevity of these encounters, however, imposes a need for simplification and reformulation of knowledge, which accentuate existing characteristics in Tibetan medicine, such as Buddhism taken here as an example, and may convey distorted or truncated ideas to their interlocutors. This article shows that the processes involved in the "translation" of Tibetan medical knowledge are not restricted to matters of language or the influence of the new market in Tibetan medicine in the region. In trying to understand these expressions of knowledge, one encounters a particular, two-pronged demand: The need to reflect on the architecture of erudite knowledge in the Tibetan world, and on the regional sociopolitical and economic dynamics. These considerations will help to place in proper perspective some assumptions on the enchantment and transformation of Tibetan medicine in similar environments.

Keywords : therapeutic knowledge, globalization, international patients, translation, Buddhism, Tibetan medicine, Ladakh 


\section{Resumen: Acentuaciones y pragmatismo. El saber médico TIBETANO CON DESTINACIÓN A LOS EXTRANJEROS}

Desde mediados de los años 1970, el desarrollo del turismo de temporada en la región himalayense de Ladakh atrae a numerosos extranjeros interesados en la medicina tibetana. Algunos practicantes de esa medicina familiarizados con el inglés aprovecharon esta ocasión para dirigirse directamente a esta nueva clientela a través de consultas clínicas o de cortas exposiciones públicas. Este artículo trata de esta manifestación local de la globalización terapéutica. Más precisamente, se interesa por el modo en el que los practicantes presentan un saber médico elaborado y complejo con la intención de hacerlo inteligible para los pacientes internacionales habitualmente profanos. Los practicantes proceden de modo pragmático esperando establecer un espacio de comunicación pero ni su saber médico ni su práctica se encuentran profundamente modificados. La brevedad de estas interacciones impone sin embargo una simplificación y una reformulación del saber que acentúa características existentes en la medicina tibetana, como el budismo y lo «religioso» tomados aquí como ejemplos, y proporciona concepciones a veces erróneas a los extranjeros. Este artículo muestra que los procesos de «traducción» de los conocimientos médicos tibetanos no se reducen a cuestiones de lenguaje $\mathrm{o}$ a la influencia del nuevo mercado de la medicina tibetana en la región. Comprender plenamente estas enunciaciones del saber requiere una doble exigencia suplementaria: hay que considerar la arquitectura del saber erudito en medio tibetano y prestar atención a las dinámicas regionales sociopolíticas y económicas. Estas consideraciones permiten interrogar más generalmente ciertas ideas admitidas sobre el encantamiento y la transformación de la medicina tibetana en contextos similares.

Palabras clave: saber terapéutico, globalización, pacientes internacionales, traducción, budismo, medicina tibetana, Ladakh 